\title{
Reliability Evaluation Model Of Industrial Internet Of Things Systems
}

\author{
Dong-Seong Kim, Hoa Tran-Dang \\ Dept. of IT Convergence Engineering, Kumoh National Institute of Technology, Gumi-Si, Republic \\ of Korea Correspnoding Author: Dong-Seong Kim. \\ Email:dskim@kumoh.ac.kr
}

\begin{abstract}
The Industrial Internet of Things (IIoT) allows digitizing manufacturing processes and increasing the digital connectivity of smart factory and industrial systems. The reliability of a system is considered as a key performance indicator that defines how accurately and perfectly the system works. Ensuring reliability in industrial IoT exposes several challenges as well as promising opportunities for advancing technologies and systematic designs such as algorithms, architectures, and devices. It depends on several factors, for example, ensuring performance, accuracy, stability, and availability. This article provides a systematic model for evaluating the reliability of IIoT systems. This model enables elucidate several open research issues regarding designing the reliable and robust systems.
\end{abstract}

\section{Introduction}

Recent years have witnessed a "connect the unconnected" vision in academia, business, industry, and society that enables millions of resource-constrained embedded devices, objects, and humans to connect together through the Internet protocol for an ubiquitous data exchange. This network of ubiquitous smart objects is called the Internet of Things (IoT) [1] which leverages the pervasive interconnectivity to collect reliable and safe real-time data and transforms it into valuable information for monitoring and controlling applications. The diverse applications that have been envisioned from the IoT paradigm to achieve an unprecedented performance improvement in productivity, efficiency, and safety can be classified into two main classes [2]. The first class is referred to as consumer IoT including smart home entertainment systems, smart meters, smart home energy management networks, which generally offer convenience, efficiency, and intelligence to the users to maximize the usage of time and resources. In the IoT-based consumer systems, the underlying data communication follows the client-server mode in which a large amount of data is periodically sent from the IoT devices to the servers to create the applications and services. Typically, the consumer IoT applications exclude emergency situations as something goes wrong during the system operations. In particular, the main feature of these applications is a ubiquitous interaction between users and IoT devices, which, in turn, enables humans to reconfigure and recover the systems in case of failures. The second class including applications from monitoring to autonomous controlling purposes in the industrial sectors is termed as Industrial IoT (IIoT) [3], [4]. In these IIoTbased systems, the data exchanges are not merely the client-server communication model but emphasize on the Machine-toMachine (M2M) communication to share valuable data. On this basis, groups of IoT devices can collaborate and work as independent networks to perform tasks. In addition, as empowered by advanced computing and communication the nodes (i.e., IoT devices) can play a role as peers on the network which are capable of making decisions as well as reporting status to the central server or other nodes. Unlike the first application class, failures and downtime in any industrial operation of IIoT-based systems can lead to highly dangerous situations or even life-threatening. Practically, the existing technologies, operation, protocols for consumer devices are completely inapplicable in the industrial systems, which require critical priorities such as safety, accuracy, and time sensitivity. Especially, the industrial automation and peer-to-peer distributed control systems heighten the demand for an extremely increased reliability since human interventions are impossible in rapid speed 
automation processes as well as "lights-out" environments. In addition, that reliability is also crucial for an economic reason. Practically, the industrial systems include a set of highly valuable assets such as machines, devices, equipment, which may provide safety-critical data generated by integrated industrial sensors. Therefore, failures in transmitting such important data reliably to the central managements may cause an outage of entire production line, damage to the factory infrastructure, or even the loss of worker lives. In such cases, a high expenditure is added for activities to overcome the troubles such as infrastructure replacement or maintenance activities. All these points mentioned above indicate strongly that reliability becomes the top priority when designing an IIoT-based system.

\section{Systematic Model of Reliability Evaluation for IIoT Systems}

According to [9-10], the reliability and efficiency of high performance IIoT systems can be evaluated through four basic fundamental quality metrics: usability, dependability, availability, and maintainability. Corresponding to the IIoT architecture, the model to evaluate the complete reliability of IIoT technological components is shown in Fig. 1, which is adopted in this paper to analyze and investigate the end-to-end reliability measurement through the constituent components of IIoT systems.

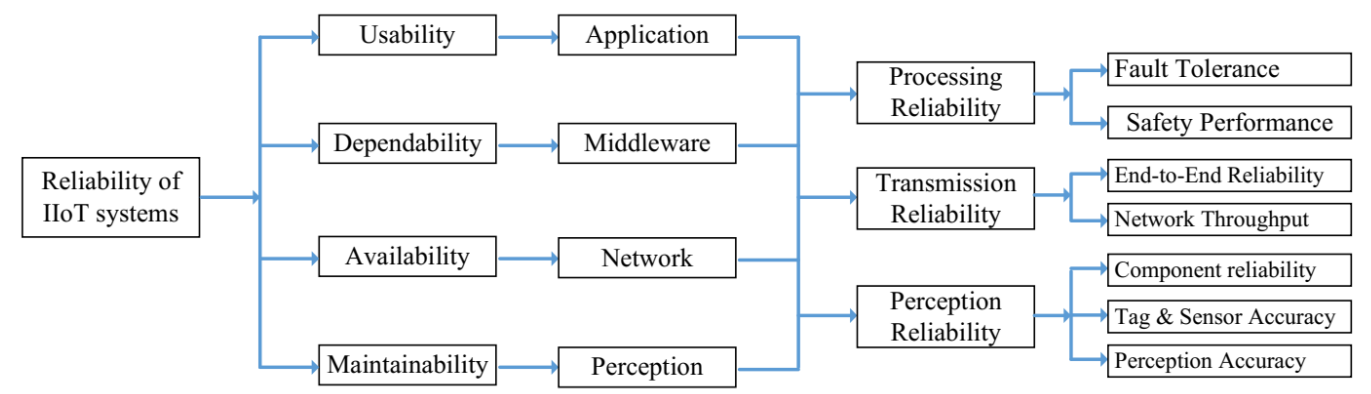

Figure 1: An evaluation model for IIoT system reliability.

The reliability of a system is a performance measurement factor that defines how accurately and perfectly the system works [11-12]. Ensuring reliability in the IIoT-based systems impose a promising challenge since it depends on several factors, for example, ensuring performance, accuracy, stability, and availability [13-14]. The challenges can be resulted from every functional block of IIoT systems such as perception, network, middleware, or application. For example, at the perception layer, imperfect sensing or identification due to hardware-related hardware can lead to an unreliable data that is the original source of systematic unreliability. The network layer exposes a big and inherent issue to the system reliability since it is vulnerable to communication errors caused by nature of wireless interferences, industrial obstacles, and environments. Therefore, to ensure total IIoT system reliability, the data-driven operations of every part of IIoT systems (e.g., data acquisition, data transmission, data processing and analysis, etc.) needs to be reliable. In addition, performanceimprovement methods and techniques are developed for supporting the highly reliable IIoT systems.

\section{References}

[1] J. Gubbi, R. Buyya, S. Marusic, and M. Palaniswami, "Internet of things (iot): A vision, architectural elements, and future directions," Future Generation Computer Systems, vol. 29, no. 7, pp. 1645 - 1660, 2013.

[2] D. Miorandi, S. Sicari, F. D. Pellegrini, and I. Chlamtac, "Internet of things: Vision, applications and research challenges," Ad Hoc Networks, vol. 10, no. 7, pp. 1497 - 1516, 2012.

[3] T. Lennvall, M. Gidlund, and J. Akerberg, "Challenges when bringing IoT into industrial automation," in 2017 IEEE AFRICON, Sep. 2017, pp. 905-910.

[4] D.-S. Kim and H. Tran-Dang, An Overview on the Industrial Internet of 
Things. Cham: Springer International Publishing, 2019, pp. 207-216.

[5] O. Gandhi, V. Agrawal, and K. Shishodia, "Reliability analysis and evaluation of systems," Reliability Engineering \& System Safety, vol. 32, no. 3, pp. 283 305, 1991.

[6] A. Birolini, Reliability Engineering: Theory and Practice, ser. Engineering online library. Springer Berlin Heidelberg, 2003.

[7] A. AB, System 800xA Solutions Handbook. ABB, January 2016.

[8] R. Billinton and R. N. Allan, System reliability evaluation using probability distributions. Boston, MA: Springer US, 1992, pp. 221-259.

[9] L. Yong-Fei and T. Li-Qin, "Comprehensive evaluation method of reliability of internet of things," in 2014 Ninth International Conference on P2P, Parallel, Grid, Cloud and Internet Computing, Nov 2014, pp. 262-266.

[10] P. Kunkun and L. Xiangong, "Reliability evaluation of coal mine internet of things," in the 2014 International
Conference on Identification, Information and Knowledge in the Internet of Things, Oct 2014, pp. 301-302.

[11] P. P. O'Connor and A. Kleyner, Practical Reliability Engineering, 5th ed. Wiley Publishing, 2012.

[12] T. Fruhwirth, L. Krammer, and W. Kastner, "Dependability demands and state of the art in the internet of things," in 2015 IEEE 20th Conference on Emerging Technologies Factory Automation (ETFA), Sep. 2015, pp.1-4.

[13] K. Bowman and B. Cline, "How the internet of things will improve reliability tracking," in 2015 Annual Reliability and Maintainability Symposium (RAMS), Jan 2015, pp. 1-5.

[14] B. Chao, X. Zhu, Q. Li, and A. Huang, "Reliability management in software requirement analysis," in 2006 IEEE International Conference on Management of Innovation and Technology, vol. 2, June 2006, pp. 1104-1107. 